\title{
L'Analyse praxéologique. Composition, ordre et articulation d'un procès
}

Praxeological analysis. Composition, order and articulation of a process

\section{Claudine de France}

\section{(2) OpenEdition}

1 Journals

Édition électronique

URL : https://journals.openedition.org/tc/5000

DOI : $10.4000 /$ tc. 5000

ISSN : 1952-420X

Éditeur

Éditions de l'EHESS

\section{Édition imprimée}

Date de publication : 30 juin 2010

Pagination : 220-222

ISSN : 0248-6016

\section{Référence électronique}

Claudine de France, "L'Analyse praxéologique. Composition, ordre et articulation d'un procès »,

Techniques \& Culture [En ligne], 54-55 | 2010, mis en ligne le 30 janvier 2013, consulté le 29 septembre 2022. URL : http://journals.openedition.org/tc/5000; DOI : https://doi.org/10.4000/tc.5000 


\section{L'ANALYSE PRAXÉOLOGIQUE}

\section{Composition, ordre et articulation d'un procès}

Comment aborder l'inextricable enchevêtrement des manifestations d'un procès technique en tenant compte simultanément de son déroulement dans le temps, de son déploiement dans l'espace, de la part respective prise par les contraintes matérielles et les obligations rituelles? Tel est l'un des objets de l'analyse praxéologique, entendue comme une méthode d'investigation des formes de l'action, et notamment de celles que rend observables le comportement technique.

\section{Les articulations temporelles d'un procès}

Étant anthropologue cinéaste, j'exposerai ici le point de vue d'un chercheur utilisant avant tout l'image animée pour étudier les activités humaines, tout en s'interrogeant constamment sur les problèmes soulevés par cet emploi, c'est-à-dire sur des questions de méthode et de mise en scène, et sur la contribution qu'apporte l'usage du cinéma à cette science de l'action qu'est la praxéologie.

Depuis 1968, j’ai été amenée à filmer en France des activités quotidiennes de ma propre société: des techniques du corps (Le Coiffeur itinérant 1972, Techniques de musculation 1973); des techniques matérielles artisanales (La Charpaigne 1968) ou domestiques (Laveuses 1970, Bazar 1980-1982). Lors des enregistrements, puis de leurs multiples examens, mon attention fut très vite attirée par la présence, ou au contraire l'absence, 
de pauses dans le travail de l'artisan, de la ménagère, du coiffeur et, plus encore, des gymnastes. Ces pauses, ou absences de pause, semblaient être les unes et les autres de nature différente, tantôt dues à une contrainte, tantôt librement introduites.

Un observateur cinéaste est naturellement sensible à ces questions, ne serait-ce que parce qu'il doit à tout moment décider du commencement, de l'interruption ou de la prolongation de son enregistrement. Faire en sorte que coïncident ou non ses propres interruptions, dans l'espace et dans le temps filmiques, avec les ruptures dans l'activité des personnes filmées, est pour lui une question vitale. De la réponse qu’apporte le cinéaste aux interrogations: où couper? quand couper? dépend en grande partie la nature du rapport qu'entretient le procès montré, sur l'image, avec le procès observable que cette image présente. Les contraintes de l'instrumentation cinématographique sont à l'origine de telles préoccupations, les caméras - et plus particulièrement celles dotées d'un moteur mécanique - obligeant bien souvent le cinéaste à introduire des ruptures totalement artificielles dans l'enregistrement, qui brisent le flux de l'action filmée.

C'est ainsi que lors du tournage de La Charpaigne ${ }^{1}$ les pesanteurs instrumentales - la nécessité de remonter le ressort du moteur de la caméra toutes les trente secondesm'ont contrainte d'interrompre à plusieurs reprises l'enregistrement alors que l'action du vannier elle-même ne souffrait pas d'interruption. Il s'agissait de moments critiques dans la fabrication de la corbeille, dite « charpaigne »: 1. le passage de la fermeture du « tour », montant circulaire constituant le bord de la corbeille, au clouage et à la ligature de cette fermeture; 2 l'enlèvement, au cours du tissage de la poignée, d'un taquet de bois provisoirement fiché dans la poignée pour ménager un trou entre les brins d'écorce de noisetier (coudrier), et son remplacement immédiat par la pointe amincie de «l'archet » central, montant principal donnant à la corbeille sa profondeur. Or, en dépit de mes incitations, le vannier refusa d'introduire une pause pour respecter ma propre interruption, justifiant cet enchaînement de gestes par des contraintes matérielles: toute pause dans son activité aurait été dommageable au procès, parce que l'obligeant à recommencer partiellement -sinon totalement - son travail antérieur. L'interruption arbitraire de l'enregistrement avait donc permis indirectement de mettre en évidence une certaine forme de chaîne,

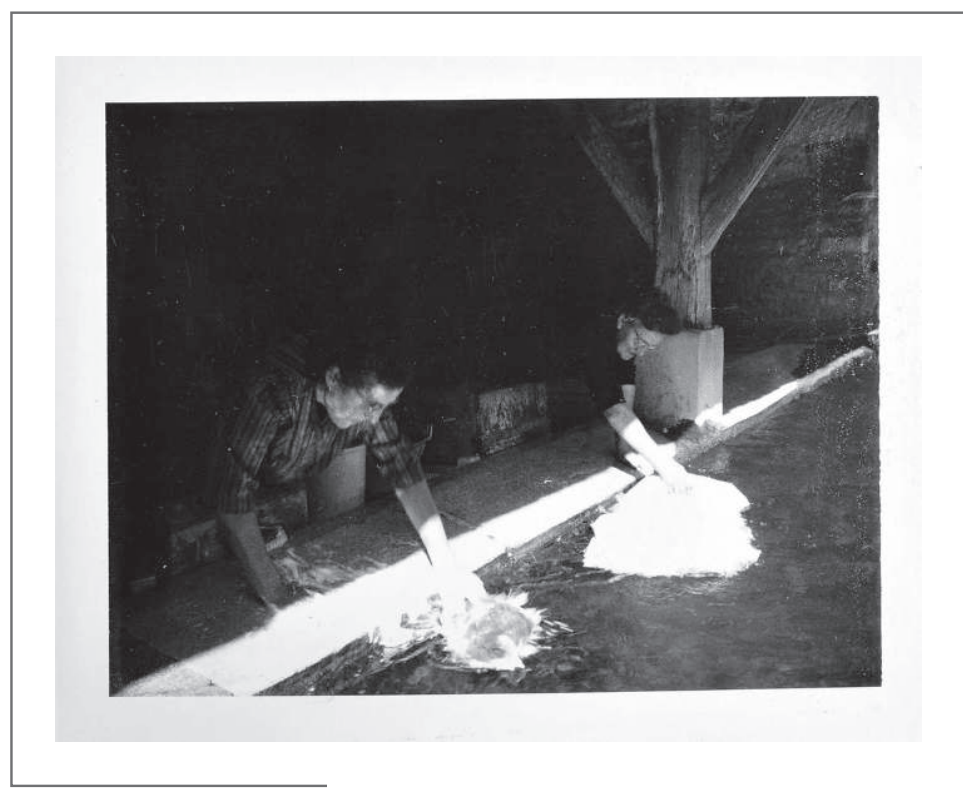
c'est-à-dire un enchaînement matériel nécessaire de gestes qui, s'il venait à être accidentellement brisé, contraignait l'agent à un retour en arrière de l'action.

En revanche, le vannier, à plusieurs reprises, marqua des pauses spontanées, soit pour bourrer et fumer sa pipe, soit pour attendre que la cinéaste reprenne son enregistrement interrompu contre son gré. Toutes ces pauses tendaient à prouver que l'activité du vannier pouvait être - en dehors des cas précédemment cités - constamment interrompue sans dommage pour le procès de fabrication de la corbeille, qui se trouvait alors simplement arrêté, puis repris là où l'avait abandonné l'artisan. On ne pouvait donc parler, à ce propos, de contrainte matérielle dans l'enchaînement temporel des gestes. 
Dès lors est apparue la nécessité de mettre l'accent sur une arthrologie des procès techniques, à partir d'une micro-analyse des articulations entre les différents moments d'un procès, examinées de proche en proche, et d'une macro-analyse s'attachant aux articulations entre de vastes ensembles de séquences d'activités. Seraient en outre soigneusement distingués les enchaînements contraignants de ceux n'ayant que l'apparence de la contrainte: d'un côté, les périodes au cours desquelles toute rupture dans l'activité de l'agent était impossible sans que ne régresse partiellement ou totalement le procès; de l'autre, les périodes qui toléraient des ruptures dans l'activité sans que régresse pour autant le procès, que ce dernier concerne ou non une fabrication.

Une notion devenait centrale: celle de « chaîne opératoire », créée par A. Leroi-Gourhan (1964,

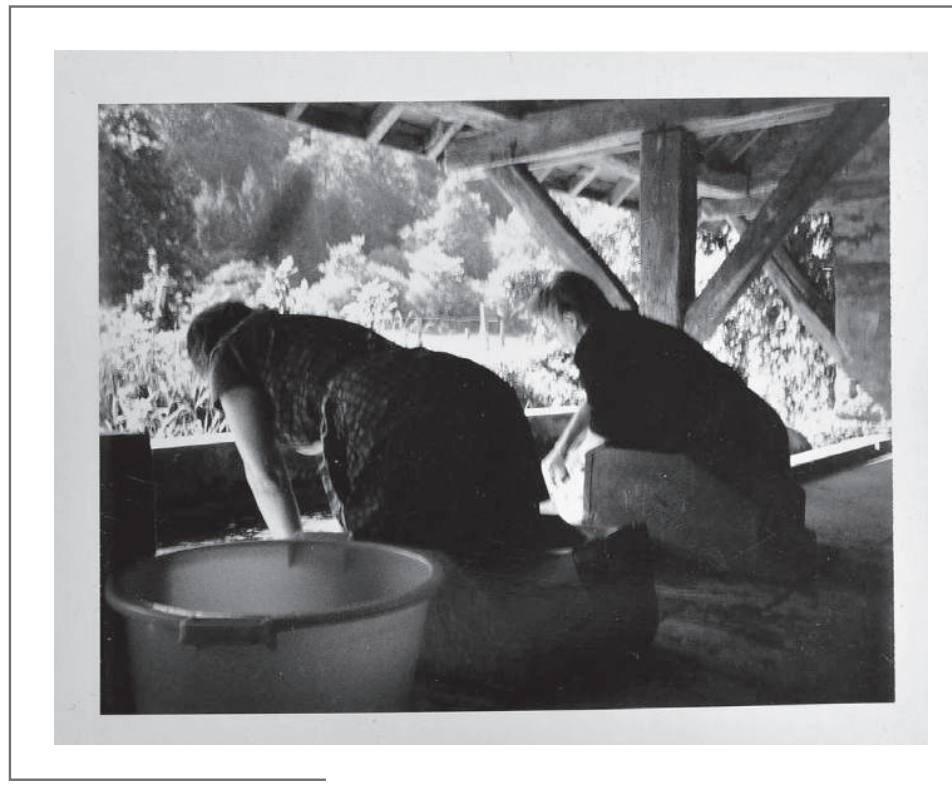
1965). Mais quel contenu et quelle extension donner à cette notion, présentée, semble-t-il, par son auteur comme l'expression d'un programme d'opérations dont les modalités d'agencement et les limites demeurent encore indéterminées? Pouvait-on concevoir de l'utiliser pour la micro-analyse de chaque procès? Et, quelle que soit son extension, ne devait-elle pas être intégrée à un plus vaste système de notions praxéologiques, parce que n'exprimant elle-même que l'un des aspects du déroulement d'un procès quelconque?

L'expérience cinématographique permit d'apporter une première réponse à ces questions, relative au contenu de la notion de chaîne. Il convenait en effet d'accorder à cette notion un sens dont la précision soit à la mesure de celle exigée de tout observateur cinéaste qui doit à chaque instant décider du début, de la fin ou de la continuation de son enregistrement en fonction du développement de l'action filmée. La rigueur de la définition dépendait ainsi en partie des contraintes de l'observation cinématographique. Dans cette perspective, la notion de chaîne, considérée jusqu'ici du seul point de vue temporel, devait s'appliquer à de strictes consécutions de gestes, d'opérations, de phases de l'action, nécessitées par la logique même du procès: par exemple, celle de la transformation de la matière en vue de l'obtention d'un produit, dans le cas d'une technique de fabrication. De ce fait, une distinction devait être opérée entre ces chaînes temporelles proprement dites - ou consécutions nécessaires-et les pseudo-chaînes temporelles - ou consécutions apparentes-, simples mises en scène de la consécution. Jonctions contingentes, les pseudo-chaînes n'étaient pas dictées par la logique du procès, leur

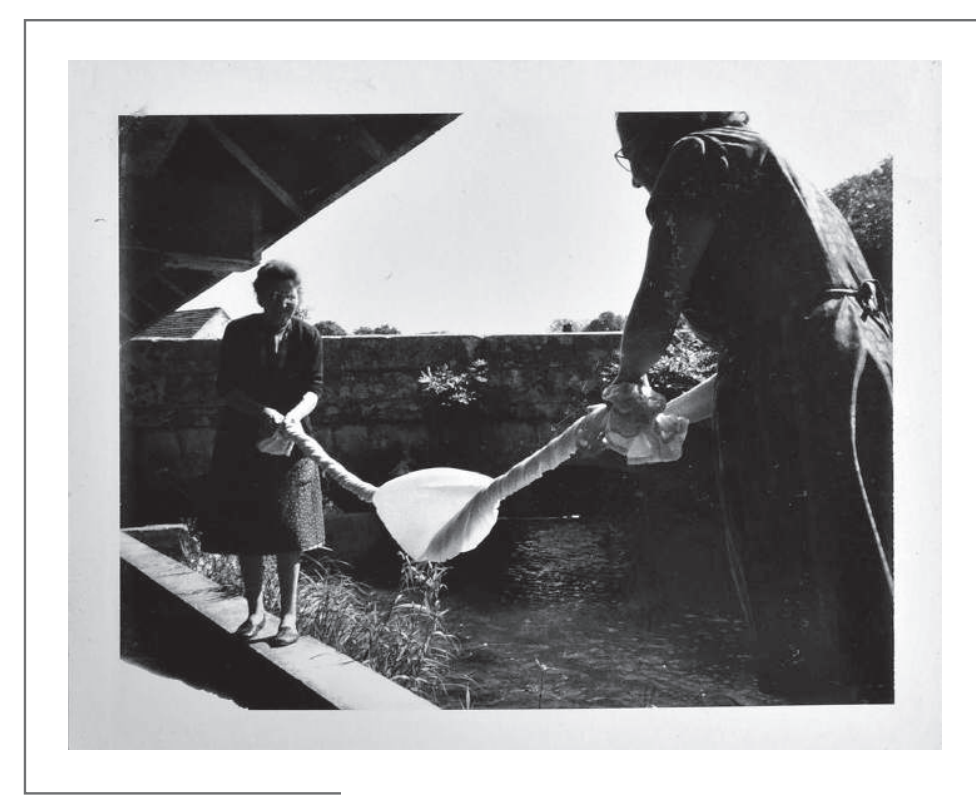




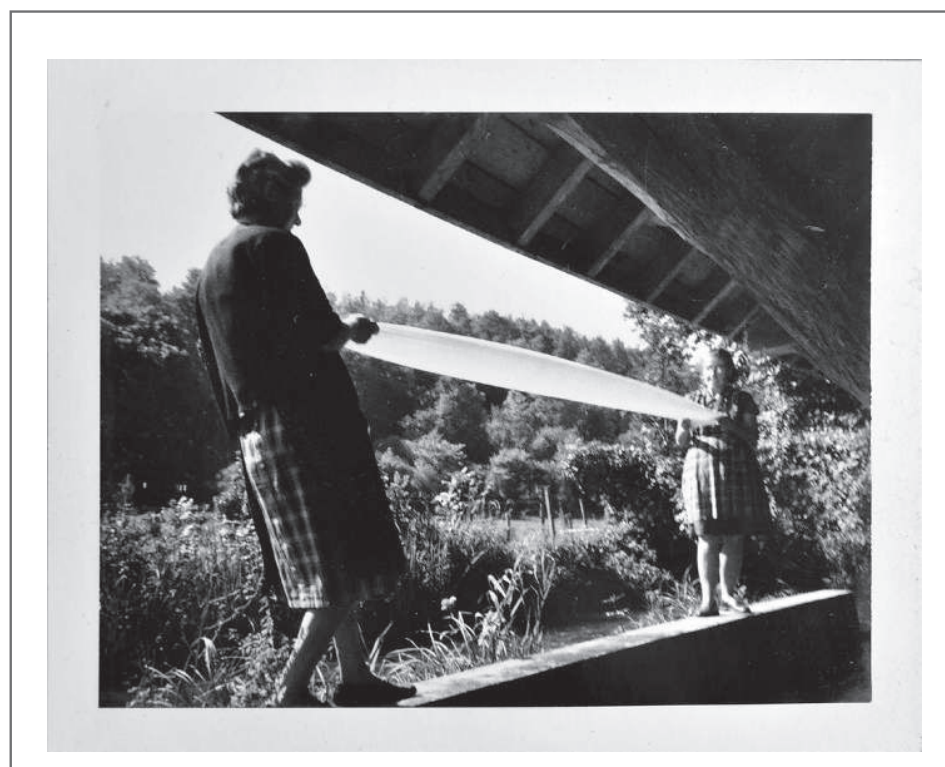

« Laveuses 》, Cl. de France, sept.1970

Photos du tournage, polaroïds N. \&B. absence éventuelle n'affectant que la durée totale du procès, non le sens de sa progression. En revanche, la nécessité interne des consécutions propres aux véritables chaînes se trouvait dévoilée lorsque, rompues accidentellement, leur interruption introduisait un double effet de régression et de répétition de l'action, l'agent se voyant dans l'obligation de recommencer, soit une phase, soit la totalité d'un procès (répétition) qui s'était défait spontanément (régression).

Entendues ainsi, les chaînes devaient être chose plus rare et plus difficile à déceler qu'il n'y apparaissait au premier abord. De même leur étendue dans le temps devait-elle être extrêmement variable. Aussi, à côté des exemples déjà cités à propos de la fabrication d'une charpaigne, concernant de très courtes périodes du procès, rencontrait-on des chaînes couvrant une longue période, parfois même la quasi-totalité du procès. Je citerai en vrac: la peinture à la fresque, le passage nécessairement immédiat de l'ensemble des opérations de capture du poisson à celles de sa conservation, la fabrication d'une poterie au tour, etc.

Quant aux pseudo-chaînes, consécutions purement apparentes puisque non nécessaires au déroulement normal du procès, leurs exemples abondaient: le continuum des gestes de La Charpaigne, à l'exception des moments critiques précédemment cités; la succession ininterrompue des gestes de lavage au lavoir (micro-analyse), telle qu'on peut la constater dans Laveuses ${ }^{2}$ et, dans ce même film, l'enchaînement immédiat, bien que facultatif, des opérations de trempage, de savonnage, de frottage, de rinçage, puis d'essorage (macro-analyse); dans Laveuses encore, la continuité des opérations de pliage d'un drap par une mère et sa fille, comme s'il s'agissait d'un geste de coopération d'un seul tenant. Or, comment qualifier, sur le plan logique, le continuum observé mais non nécessaire, de manière à le distinguer des véritables chaînes? Puisque cet enchaînement apparent pouvait être librement interrompu, se cachaient derrière lui de simples suites de gestes, d'opérations ou de phases de l'action, dont la consécution était contingente. Aussi ai-je proposé de qualifier de suite, lato sensu, toute succession revêtant tantôt la forme d'une pseudo-chaîne, tantôt celle d'une absence de chaîne, en considérant toutefois cette dernière forme comme seule véritable suite, stricto sensu, parce que telle sur le double plan logique et scénographique (coïncidence entre possibilité logique et mise en scène).

Chaînes et pseudo-chaînes offraient toutes en commun l'apparence de la consécution, forme temporelle de la jonction. Elles s'opposaient ainsi en bloc, sur le plan scénographique, aux actions non consécutives parce qu'interrompues par des pauses -les unes nécessaires, les autres contingentes-, et dont le trait commun était d'offrir l'apparence de la séparation temporelle.

Séparations nécessaires, les pauses obligées affectent l'activité de l'agent sans interrompre le déroulement du procès ${ }^{3}$, dont elles assurent la progression. Aussi ai-je proposé de les qualifier de chaînes invisibles, voulant ainsi mettre l'accent sur leur fonction de 


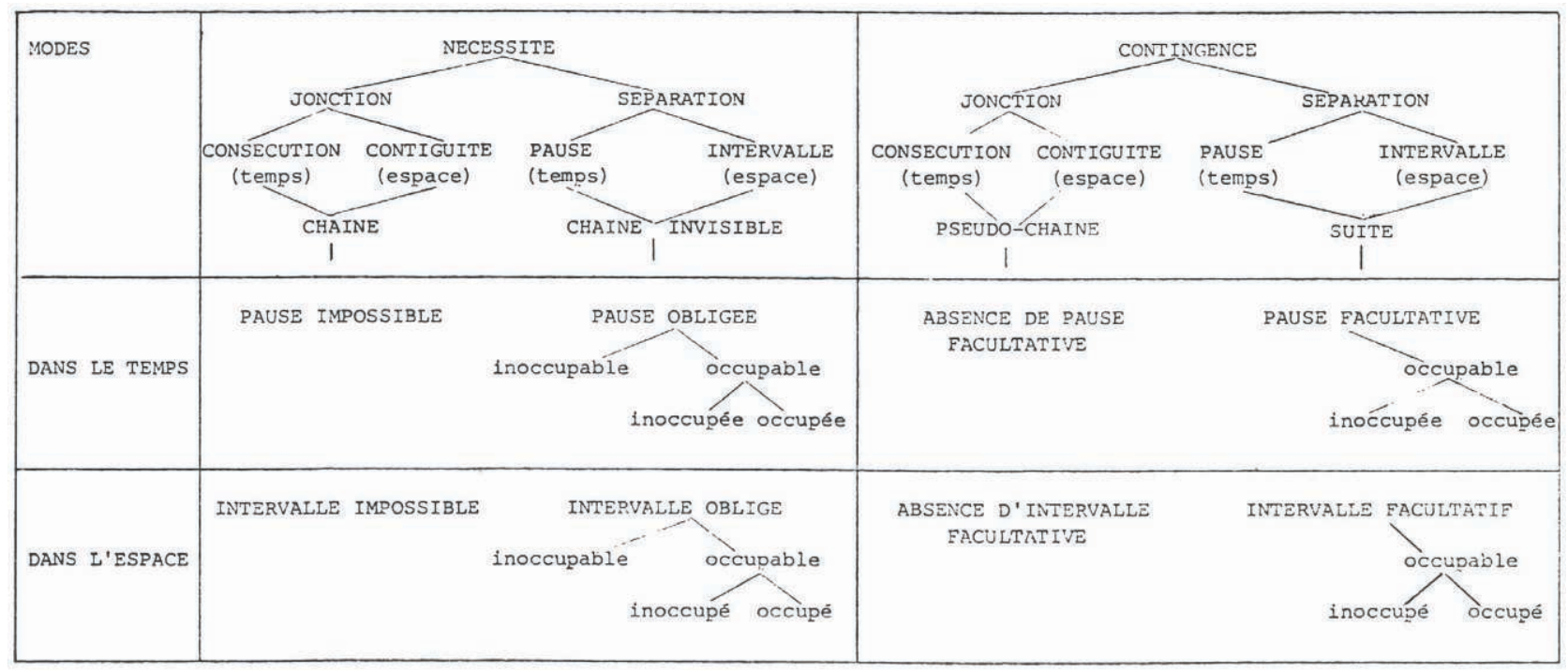

liaison nécessaire entre des moments du procès, malgré une cessation provisoire de l'activité des agents. De telles pauses se rencontrent fréquemment dans les procès de type culinaire: par exemple l'interruption qui permet au couscous de gonfler, au rôti de cuire; ou encore, dans les travaux relatifs au bâtiment: par exemple, la pause qui laisse à une première couche de peinture le temps de sécher avant que ne soit étendue une seconde couche, au ciment celui de durcir, etc.

Exact pendant logique des chaînes en ce que sans elles, le procès se trouverait compromis, les chaînes invisibles offrent des pauses obligées dans l'activité de l'agent qui, dans certains cas sont occupables - qu'elles soient ou non occupées -, dans d'autres, inoccupables par des activités secondaires, ou étrangères au procès. Je ne m'attarderai pas sur ce point dans le cadre de cet exposé (Fig. 1). C'est en enregistrant, puis en examinant les images de Techniques de musculation ${ }^{4}$ que m'est apparue de la manière la plus frappante l'importance de ces pauses obligées, qui offrent l'illusion d'un arrêt du procès. Ainsi, la séance consacrée aux exercices de musculation offrait toutes les apparences d'un procès fréquemment interrompu, alors qu'il ne s'agissait que de pauses passagères dans l'activité des gymnastes, au cours desquelles s'accomplissait la récupération énergétique du corps, phase indispensable du procès. Or, ces pauses, lors desquelles les gymnastes bavardaient assis, ou déambulaient en flânant, occupaient la plus grande partie de la séance de musculation libre, donnant à l'observateur l'impression qu'il ne se passait rien. Autrement dit, la mise en scène propre de ces pauses obligées de l'activité, non directement productives, est à l'origine de l'illusion selon laquelle le procès lui-même a cessé. Ainsi s'explique-t-on mieux qu'elles figurent rarement dans les films, notamment dans les reportages sportifs de la télévision. Quant aux séparations contingentes, figurées par des pauses observées bien que facultatives, elles relient une suite d'actions, stricto sensu dont j'ai cité un exemple: la pause libre du vannier de La Charpaigne au cours du tissage de la corbeille, mise à profit (occupée) pour bourrer, puis fumer sa pipe. Lorsque ce type de pause intervient dans l'activité de l'agent, qu'elle soit ou non occupée par des activités secondaires ou étrangères au procès - car elle est toujours occupable-, ce 
dernier voit son cours interrompu sans dommage. Il demeure en l'état, comme figé en un instantané photographique. Grâce aux pauses contingentes proposées par les suites, l'homme se libère quelque peu du tissu serré des gestes machinaux. Nombreuses sont les activités humaines qui les tolèrent, au contraire des véritables chaînes. Les notions de chaîne et de suite ayant été définies l'une par rapport à l'autre, s'est posée la question de leur extension. Le champ d'application des chaînes et des suites devait-il se limiter au registre temporel, c'est-à-dire au seul déroulement des actions, ou bien s'étendre au registre spatial, relatif à leur déploiement? De plus, ces notions ne devaient-elles concerner que les contraintes et les options propres aux procès d'ordre physique, matériel, ou bien ne pouvaient-elles également exprimer obligations et options rituelles lors de procès, soit quelconques, soit dominés par l'observance de normes et de règles rituelles, en fonction des valeurs propres au groupe ou à la société tout entière? Enfin, ne pouvait-on étendre une telle opposition, conçue dans l'esprit d'une arthrologie des formes de l'action, aux autres modes ou relations d'agencement des procès, tels que la composition (relations de co-présence entre les agents - lato sensu- ou les phases de l'action) et l'ordre (relations d'orientation entre les agents de l'action; de succession ou de simultanéité entre les phases de l'action)? Les réponses progressivement apportées à cet ensemble de questions ont eu pour conséquence l'ébauche d'un système d'analyse des relations praxéologiques. Si la notion de chaîne se situait au point de départ de cette réflexion, elle devenait, une fois le système mis en place, le résultat d'un entrecroisement de notions telles que: l'espace ou le temps; la composition, l'ordre, l'articulation; la nécessité ou la contingence; le physique ou le rituel (Fig. 2).

Figure 2

Système des relations praxéologiques.

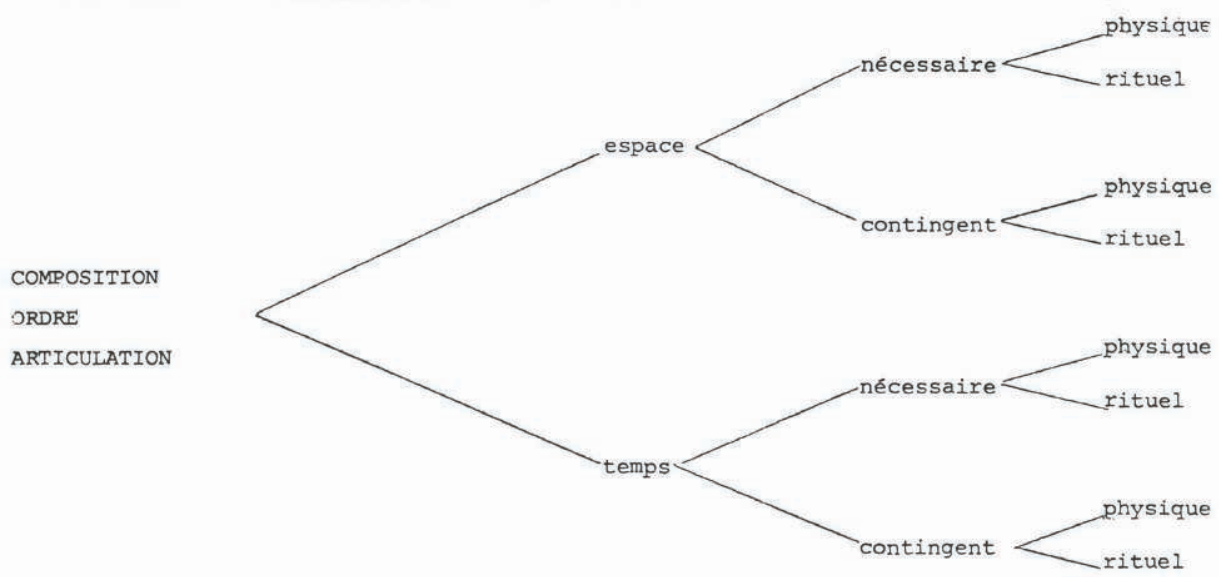

\section{Symétrie praxéologique de l'espace et du temps}

Un examen plus poussé des films a fait apparaître la possibilité d'étendre, de façon quasi-symétrique, la grille d'analyse des articulations temporelles aux articulations dans 
l'espace des éléments, ou agents lato sensu, de l'action. Par espace est ici entendu le mode de déploiement, dans le milieu, de ces éléments qui participent de façon directe ou marginale à l'action: agents humains, instruments, objets, etc.

C'est ainsi qu'aux chaînes temporelles semblent correspondre des chaînes spatiales, les jonctions ou consécutions nécessaires entre les actions (temps) ayant pour pendant des jonctions ou contiguités également nécessaires entre les éléments de l'action (espace): corps, instruments, objets. Par exemple, le film Laveuses montre de manière ostensible, dans diverses séquences, que pour plier ou essorer un drap en coopération, le contact

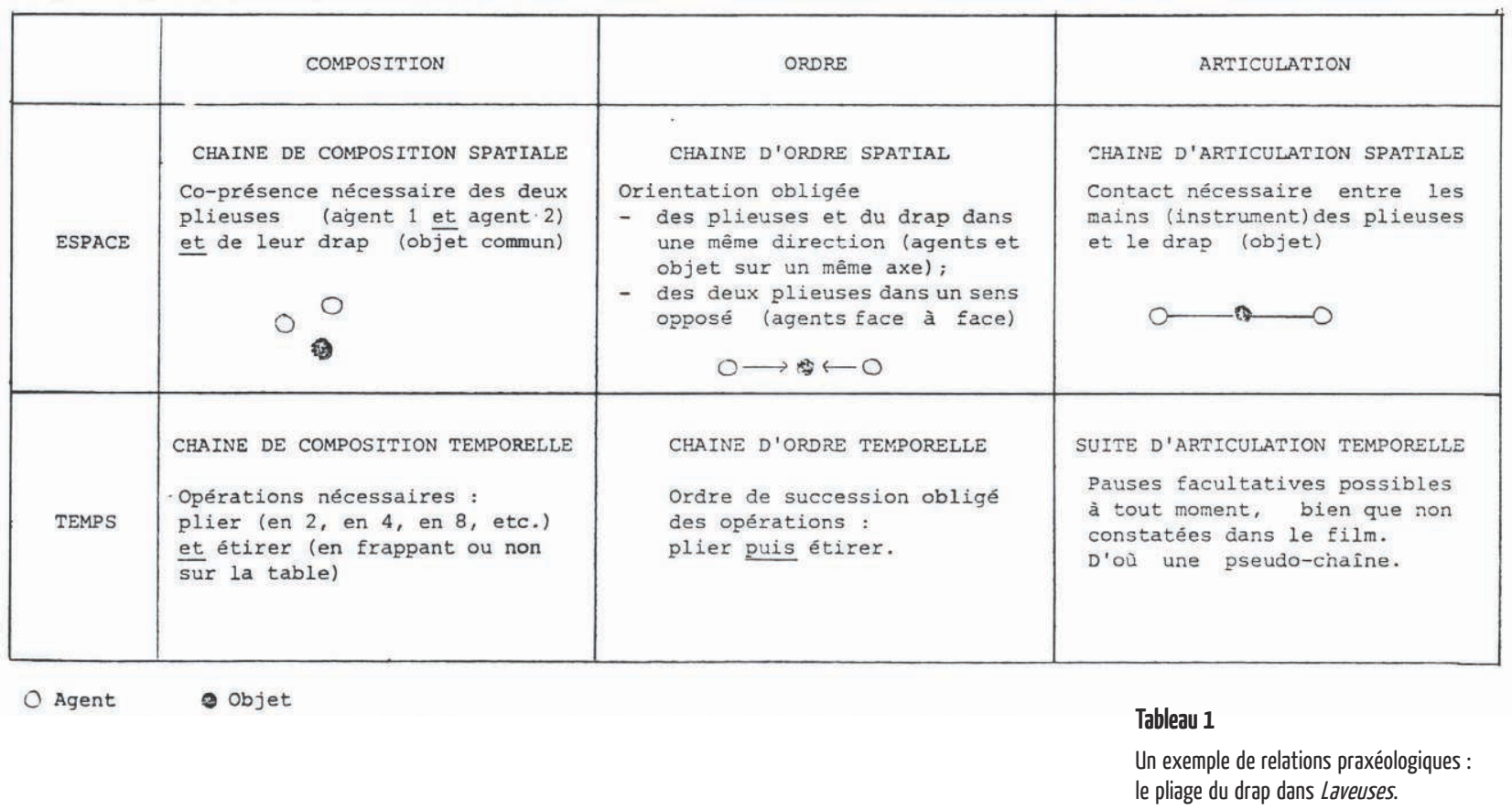

des mains des laveuses (instrument) avec le drap (objet) est presque en permanence indispensable pour la bonne marche du procès. Les mains exercent en effet constamment une double fonction instrumentale de support et de transformation de l'objet (Tableau 1).

De même correspondent aux pseudo-chaînes temporelles des pseudo-chaînes spatiales, les jonctions ou consécutions contingentes entre les actions ayant pour pendant des jonctions ou contiguités contingentes - bien qu’observées - entre les éléments physiques de l'action, qu'il s'agisse des corps, des instruments ou des objets. Ainsi dans Laveuses existent de temps à autre, à côté des contacts obligés précédemment cités, des contacts furtifs et accidentels entre les doigts des deux repasseuses occupées à plier un drap, qui n'ont aucune incidence sur le développement du procès dans son ensemble. Il en va de même dans La Charpaigne lorsque, au cours de la phase de tissage de la corbeille, le chat du vannier joue avec l'extrémité d'une tige d'écorce ("lanière ») que l'artisan est en train de manipuler.

La symétrie remarquée entre les jonctions s'observe également entre les séparations. Aux chaînes invisibles temporelles font pendant des chaînes invisibles spatiales, les pauses obligées dans le déroulement de l'activité ayant leur correspondance dans l'espace, sous 
la forme d'intervalles - ou écarts-nécessaires entre les corps, les instruments, les objets, entre les uns et les autres. Par exemple dans la séquence de Laveuses située au lavoir, l'écart entre les corps des femmes agenouillées sur la pierre au bord de la rivière, considéré du point de vue qualitatif et non quantitatif, est un intervalle obligé, de caractère fonctionnel. Il est en effet nécessaire au déploiement des gestes de lavage de chacune, et par conséquent, au déroulement correct du procès. De même dans Le coiffeur itinérant ${ }^{5}$, le coiffeur prend soin de toujours ménager un intervalle entre ses instruments de travail disposés sur la table du café où il est de passage (ciseaux, tondeuse, peigne, rasoir), afin de faciliter leur préhension lors des changements d'instruments au cours d'une coupe de cheveux. Les agents, ou les instruments de travail, et l'intervalle qui les sépare (corps des laveuses d'un côté, outils du coiffeur de l'autre), forment ensemble une chaîne invisible, parce que l'absence de contact entre eux est indispensable à leur alignement et, plus généralement, au développement du procès (ici le lavage du linge, là la coupe de cheveux).

À l'image de ce qui se produit dans le temps, les séparations dans l'espace peuvent être purement contingentes, parce qu'observées mais facultatives, comme le sont certaines pauses dans l'activité. C'est dire qu'aux suites temporelles correspondent des suites spatiales mettant en présence des éléments de l'action qui n'entrent pas en contact les uns avec les autres, sans que cet écart change de manière significative la marche du procès. Ainsi m'est-il arrivé d'observer, au cours de la préparation de Laveuses, que lors du repassage, les piles de linge repassé parfois se touchaient (pseudo-chaînes), parfois ne se touchaient pas (suite), sans que le déroulement du repassage en soit affecté. Il en allait de même des bassines, brosses et battoirs des laveuses, déposés auprès d'elles sur la pierre, qui chez certaines se trouvaient séparés par un léger intervalle (suite), chez d'autres formaient un bloc compact (pseudo-chaînes).

Comme les pauses, les intervalles tolèrent ou non une occupation. Certains peuvent être traversés (occupables) sans dommage pour le procès et, plus particulièrement pour les agents; d'autres ne sont pas traversables (inoccupables). Parmi les intervalles occupables, certains sont effectivement occupés, d'autres ne le sont pas (Tabl. 1). Sans vouloir m'attarder sur la question de l'occupabilité des intervalles dans le cadre de cet exposé, je souhaiterais toutefois signaler son importance en ce qui concerne toute stratégie de l'observation filmique.

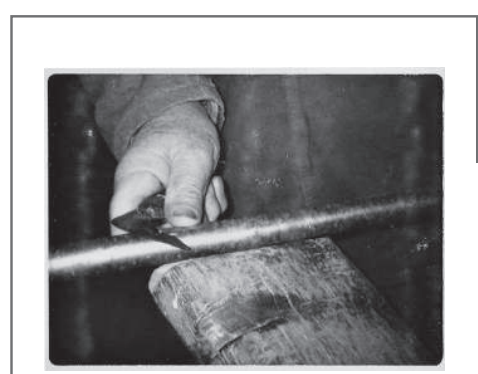

« La Charpaigne » série 1, Cl. de France, nov. 1968

Photos du tournage, polaroïds N.\&B.

\section{Chaîne et suite de composition}

Ladaptation de la matrice des articulations temporelles aux articulations spatiales, fondée sur la reconnaissance d'une symétrie praxéologique entre espace et temps, permettait d'envisager par la suite l'étude de la coordination entre actions (ou phases de l'action) et agents de l'action (ou éléments), aux différents niveaux de développement d'un procès.

Toutefois, cette première extension des notions de chaînes et de suite rencontrait des difficultés. En effet, l'accent étant mis sur les relations d'articulation entre les actions ou les agents de l'action, demeuraient encore obscures les relations de composition et d'ordre, autrement dit, ce dont étaient composés, et la manière dont s'ordonnaient respectivement, dans l'espace et dans le temps, actions et agents de l'action. Les trois modes de relations: composition, ordre et articulation n'ayant pas été posés comme tels et dissociés en esprit, 
de fréquentes confusions intervenaient au cours de l'analyse des articulations, entre les manifestations des uns et des autres. C'est ainsi qu'étaient parfois abusivement considérées comme des chaînes de consécution, ou chaînes temporelles, de simples suites temporelles d'articulation dont les formes apparentes de concaténation avaient en vérité leur origine dans des relations de composition ou d'ordre. J'en donnerai plus loin un exemple.

Il convenait donc, dans une première étape, de dissocier les manifestations des trois modes d'agencement, inséparables dans les faits; et d'examiner dans une seconde étape les possibilités d'extension, ou plus exactement d'adaptation partielle, sinon totale, de la matrice conçue pour les seules articulations, aux deux autres modes d'agencement des procès: les relations de composition et d'ordre.

Par composition, il faut entendre les relations de complémentarité - ou de co-présencedans l'espace, entre les éléments efficients ou marginaux du procès tels que les agents humains, les instruments, les objets (composition spatiale); et de complémentarité - ou de co-présence-dans le temps, entre les actions de ce même procès, qu'il s'agisse des gestes, des opérations ou des phases (composition temporelle). La composition correspond en quelque sorte à l'inventaire des ingrédients d'un procès, incluant êtres animés et inanimés, actions et choses, sans préjuger de la manière dont ils se disposent les uns par rapport aux autres, dans l'espace et dans le temps. C'est dire que ne sont pas pris en compte leur ordonnancement ni leur articulation.

\section{$[\ldots]$}

Certains éléments, ou agents de l'action, certaines actions, qui composent le procès dans l'espace et dans le temps, sont indispensables à son déploiement et son déroulement; d'autres sont facultatifs. Aussi peut-on voir dans les relations de coprésence nécessaires entre les éléments, ou entre les actions du procès, de véritables chaînes de composition, les unes spatiales, les autres temporelles.

C'est ainsi que dans Laveuses, le pliage du drap exige pour s'accomplir la présence d'éléments tels que les deux agents humains (la mère et la fille), leurs instruments corporels (les mains), et l'objet commun (le drap), le tout constituant une chaîne spatiale de composition. Mais le pliage exige aussi la présence d'au moins deux opérations: plier et étirer, qui constituent une chaîne temporelle de composition (Tabl. 1).

De même peut-on voir dans les relations de co-présence contingente entre les éléments, entre les actions d'un procès, de simples suites de composition, les unes spatiales, les autres temporelles. Par exemple, dans la séquence de Laveuses consacrée au lavoir, la présence du battoir et du paquet de lessive aux côtés de l'une des laveuses est contingente, car cette dernière ne se servira à aucun moment de l'un ou de l'autre de ces éléments du dispositif de travail. Ils forment donc avec elle une suite spatiale de composition.

Bien souvent les éléments contingents de l'espace contribuent à créer l'ambiance d'un procès. Constituant le milieu marginal, ils enveloppent l'action principale d'un réseau vivant ou matériel de manifestations visuelles et sonores. Tel est, semble-t-il, le cas des éléments marginaux de la remise du vannier, dans La Charpaigne, parmi lesquels figure son chat, les vieux paniers déjà tissés et suspendus ici ou là, les anciens outils de son grand-père posés sur un établi à l'arrière-plan, etc. ${ }^{6}$

En définitive, l'analyse des relations de composition en termes de chaînes et de suites, à l'image de ce qui fut fait pour les articulations, repose sur la possibilité de considérer la complémentarité - ou la co-présence - des agents de l'action, ou des actions, comme l'équivalent des jonctions entre ces mêmes constituants du procès dans le cas des articulations. J'en resterai là pour l'instant.
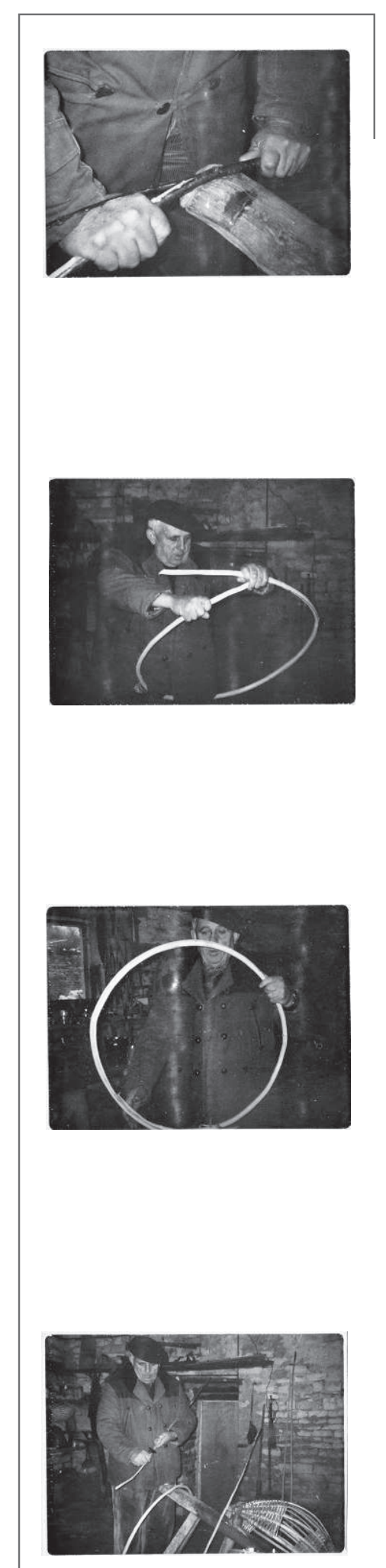

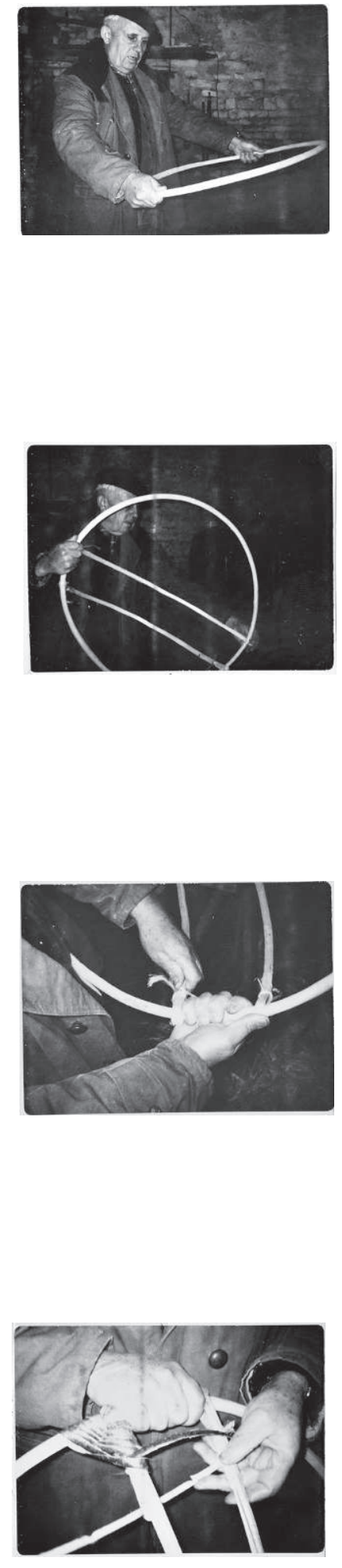

Avant d'aborder la question des relations d'ordre, je dois indiquer toutefois que dans la perspective d'une praxéologie générale, l'analyse des relations de composition doit tenir compte des niveaux de détermination des contraintes et des options du procès. Car ce qui apparaîtra comme une chaîne de composition (contrainte) à un certain niveau d'appréhension, par exemple celui de la fonction d'un dispositif spatial, se révélera une suite de composition (option) à un autre niveau, par exemple celui de la forme particulière de ce même dispositif. Le vannier de La Charpaigne et son banc à planer de tonnelier en fournissent l'illustration. En tant que support fonctionnel de l'objet qu'est la corbeille en cours de fabrication, le banc à planer constituerait avec le vannier une chaîne « fonctionnelle » de composition spatiale. Mais en tant que forme particulière de support, substitut externe et matériel du support corporel des vanniers, les genoux, ce même banc à planer créerait avec l'artisan filmé une-simple suite « formelle » de composition spatiale ${ }^{7}$.

\section{Ordre libre, ordre obligé}

Sur quelles bases concevoir à présent l'application aux relations d'ordre des principes d'analyse adoptés à propos des relations d'articulation, puis de composition? En premier lieu, qu'entendre par ordre?

En termes de praxéologie, l'ordre peut être défini comme l'ensemble des relations d'orientation entre les éléments - agents de l'action - qui composent le procès dans l'espace: agents humains, instruments, objets. Cet ordre spatial est indépendant des relations de contiguité ou de séparation, autrement dit des contacts ou des intervalles, entre les éléments concernés. De façon symétrique, l'ordre peut être défini, dans le temps, comme l'ensemble des relations de succession ou de simultanéité entre les actions qui composent le procès: gestes, opérations ou phases. Ainsi défini, l'ordre temporel est à son tour indépendant des relations de consécution ou de non-consécution entre les actions, autrement dit de l'absence ou de la présence des pauses.

Comme l'ont été jusqu'ici la composition et l'articulation, l'ordre peut être envisagé selon le mode de la nécessité ou de la contingence praxéologique. Aussi peut-on concevoir d'étendre les notions de chaîne et de suite aux relations d'orientation (espace), de succession et de simultanéité (temps), qui constituent l'équivalent de la jonction (articulations) et de la coprésence (composition). L'ordre des actions est, en effet, dans certains procès, obligé (nécessité), dans d'autres, libre (contingence), même si extrêmement probable. Il en va de même de l'orientation des agents de l'action, tantôt obligée, tantôt libre.

L'ordre temporel obligé implique, du moins dans le cas de la succession, l'impossibilité, pour l'agent humain, de permuter les actions d'un procès, sans porter préjudice à son développement. Par exemple, lors du pliage d'un drap dans Laveuses, les opérations de pliage proprement dit et d'étirage obéissent à un ordre successif obligé sans le respect duquel le drap ne parviendra pas à être correctement plié (Tabl. 1). La succession obligée de ces opérations forme une véritable chaîne d'ordre temporelle. On observe une chaîne d'ordre équivalente dans la séquence du même film se déroulant au lavoir. Le procès de lavage d'une même pièce de linge implique l'ordre successif suivant entre les diverses opérations qui le composent: trempage, savonnage, frottage, rinçage, essorage. Transgresser cet ordre rendrait absurde l'action des laveuses et irait à l'encontre du résultat recherché ${ }^{8}$. 
Lordre temporel libre en revanche, traduit la possibilité, pour l'agent humain, d'introduire une permutation dans les actions, à l'intérieur d'une même opération, ou entre des opérations successives, sans compromettre la bonne marche du procès et l'obtention du résultat final. Bien des procès domestiques s'offrent ainsi comme une simple suite temporelle d'ordonnancement entre diverses tâches. C'est ce qu'illustre le film d'Ettore Scola Une journée particulière, dans lequel l'héroïne (interprétée par Sophia Loren) doit effectuer un certain nombre de travaux ménagers dans la même journée (chaîne de composition), mais dans un ordre librement décidé par elle (suite d'ordre) : laver la vaisselle, faire les lits, étendre le linge, etc. La relative liberté dont jouit cette mère de famille, dans l'ordonnancement -comme d'ailleurs dans l'articulation - des tâches ménagères, est à la base du scénario, puisqu'elle lui permet précisément de nouer une brève et étrange idylle avec son voisin (incarné par Marcello Mastroianni), tout en accomplissant malgré tout des travaux qui lui sont imposés avant le retour du mari et des enfants.

De son côté, l'ordre spatial impose, lorsqu'il est obligé, une disposition orientée des éléments de l'action les uns par rapport aux autres, dont le dérangement compromet également la marche du procès. C'est ainsi que les deux repasseuses de Laveuses forment entre elles, et avec le drap qu'elles plient, une chaîne d'ordre spatial, car elles doivent, pour coopérer correctement, être orientées face à face, aux deux extrémités de l'axe de coopération. Toute autre orientation réciproque est exclue, parce qu'elle compromettrait le procès (Tabl. 1). De même, au lavoir, les trois laveuses ne peuvent être disposées que côte à côte et face à l'eau. Cette chaîne d'ordre spatial est indépendante du fait que les laveuses doivent ménager entre elles un intervalle pour ne pas se gêner dans leur travail (chaîne invisible d'articulation).

\section{Autonomie des trois modes de relations praxéologiques}

À l'opposé de l'ordre spatial obligé, l'ordre libre se traduit par des orientations entre les éléments de l'action, dont l'éventuel dérangement n'affecte pas véritablement le développement du procès. Ainsi, les bacs et autres ustensiles entassés autour des laveuses sur le sol du lavoir, tandis qu'elles sont agenouillées sur leur support de bois (« carrosse »), présentent une disposition librement orientée. L'ordre spatial, contingent, est dû en partie au hasard, et variable d'une laveuse à l'autre. Les ustensiles forment donc entre eux, et avec leur propriétaire, une suite d'ordre spatial. Leur libre orientation est indépendante du fait que certains d'entre eux (brosse, paquet de lessive, battoir) sont contingents quant à la composition, d'autres (bacs) nécessaires. De même est-elle indépendante de leur accolement ou de leur séparation, variables, non seulement d'une laveuse à l'autre, mais d'un lavage à l'autre pour une même laveuse (suite d'articulation).

La mise à l'épreuve des catégories de l'analyse praxéologique ici proposées a fait apparaître, après une longue période de tâtonnements, qu'une réelle autonomie existait entre les contraintes de composition, d'ordre et d'articulation, contrairement à ce qu'aurait laissé supposer l'indissociabilité, dans les faits, de leurs manifestations. L'indépendance relative des trois modes d'agencement s'accompagnait de celle des registres spatial et temporel, à l'intérieur d'un même mode.
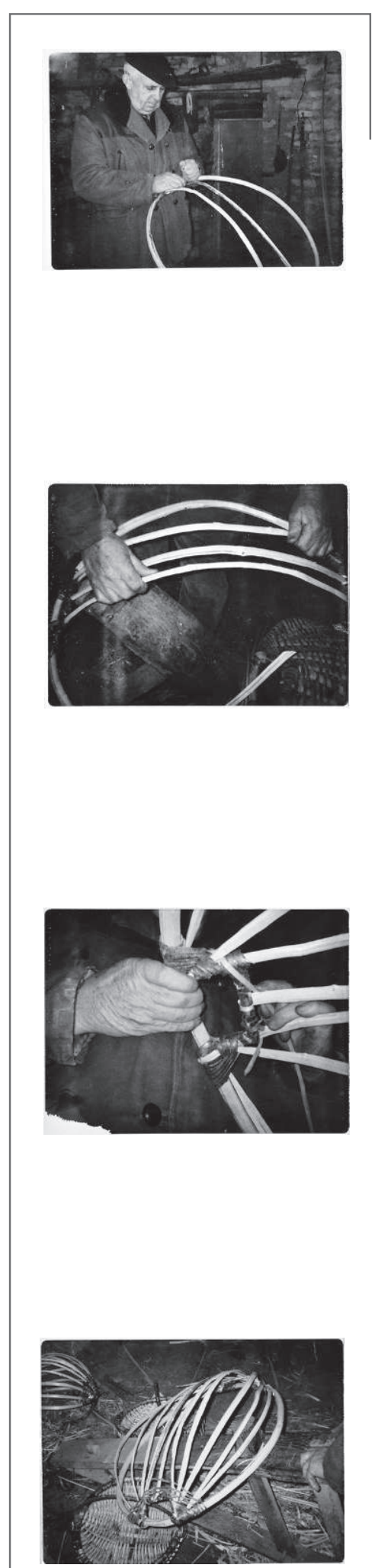

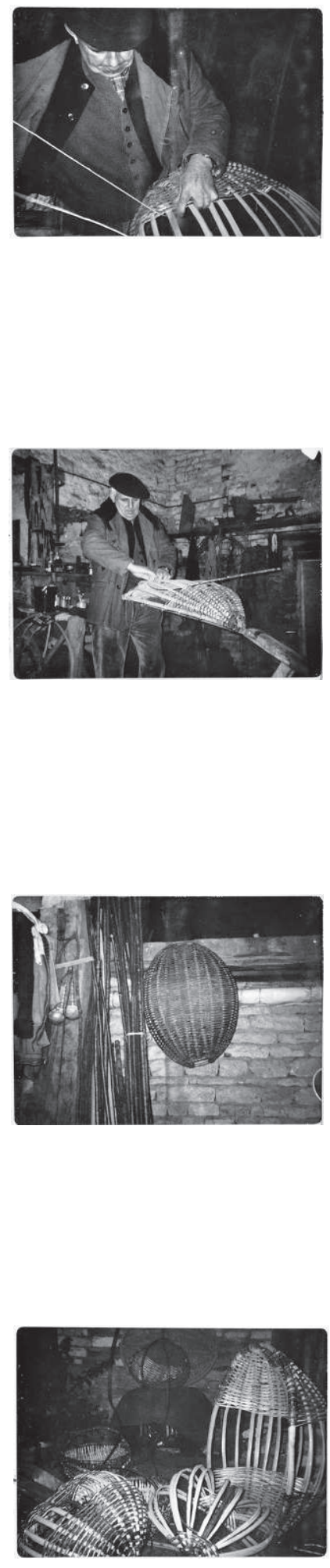

Lautonomie des trois modes peut être exprimée de la manière suivante: pour un même procès, une chaîne de composition entre des éléments de l'action, ou entre des actions de ce procès, n'implique pas nécessairement une chaîne d'ordre et/ou d'articulation entre ces mêmes constituants; réciproquement, une chaîne d'ordre n'implique pas nécessairement une chaîne d'articulation et/ou de composition, de même qu'une chaîne d'articulation n'implique pas nécessairement une chaîne de composition et/ou d'ordre. Autrement dit, il n'existe pas de relations d'implication nécessaire, simple ou réciproque, entre les trois modes d'agencement, des contraintes dans un ou deux de ces modes n'impliquant pas nécessairement des contraintes dans le ou les modes restants.

Que des contraintes dans la composition et l'ordre n'en impliquent pas dans l'articulation est relativement aisé à comprendre. On a vu, par exemple, que la double chaîne temporelle de composition et d'ordre qui, dans Laveuses, relie les opérations de trempage, savonnage, frottage, rinçage, essorage au lavoir, n'implique aucunement l'existence, malgré les apparences, d'une chaîne d'articulation temporelle entre ces mêmes opérations.

On admet moins aisément, en revanche, qu'une chaîne d'articulation, notamment entre les actions successives d'un procès, n'implique pas nécessairement l'existence d'une chaîne de composition entre ces mêmes constituants, à plus forte raison si elle s'accompagne d'une chaîne d'ordre. C'est pourtant ce qui se produit lorsque deux opérations successives doivent être nécessairement consécutives (chaîne d'articulation temporelle), bien que la présence de la seconde soit optionnelle, contingente -même s'il s'agit d'une option restreinte à deux possibilités seulement (suite de composition). Par exemple, dans la séquence de repassage de Laveuses, une fois terminée l'opération de repassage de la première d'une série de pièces de linge, deux possibilités s'offrent à la ménagère. Soit déposer aussitôt le fer sur son support isolant sans déployer une nouvelle pièce de linge froissé sur la table, soit déployer immédiatement, et rapidement, une nouvelle pièce de linge froissé, tandis que le fer repose encore sur la surface inflammable de repassage. Autrement dit, la repasseuse se trouve devant une option de composition temporelle: l'action de poser le fer sur son support isolant, ou bien celle de déployer rapidement une nouvelle pièce de linge. Cependant, quelle que soit l'action choisie, celle-ci devra être immédiate, consécutive à la fin du premier repassage, sous peine de brûler le tapis, témoignant ainsi d'une contrainte d'articulation temporelle.

Entre l'espace et le temps, au sein d'un même mode d'agencement, on observe une semblable autonomie. C'est ce que met en évidence, dans le même film, la séquence de pliage des draps. Les contraintes s'y exercent à tous les niveaux de la composition et de l'ordre, mais au seul niveau de l'espace en ce qui regarde l'articulation. En effet, pour que s'effectue le procès, les deux plieuses sont contraintes à certaines opérations (chaîne de composition temporelle) strictement ordonnées (chaîne d'ordre temporelle), à l'aide d'un dispositif de coopération inévitable (chaîne de composition spatiale), rigoureusement orienté (chaîne d'ordre spatiale); enfin, leurs mains ne doivent en aucun cas perdre le contact avec l'objet commun, le drap (chaîne d'articulation spatiale). Elles jouissent toutefois d'une liberté: celle de marquer à tout instant une pause dans le déroulement de l'activité (suite d'articulation temporelle), sans devoir pour autant recommencer partiellement ou totalement le travail ${ }^{9}$. Une chaîne d'articulation spatiale entre les éléments du dispositif d'action n'implique donc pas nécessairement la présence d'une chaîne d'articulation temporelle entre les actions du même procès (Tabl. 1).

Le pliage d'un drap par les deux laveuses revêt, en raison de leur coopération dans le simultané, des aspects plus complexes sur lesquels je n’ai pas insisté. Ainsi, dans 
les relations d'ordre spatial, la chaîne d'orientation entre les deux agents et leur objet commun est multiple; elle concerne à la fois la direction de leur interaction (les deux plieuses doivent être situées sur le même axe de déploiement que le drap) et le sens de cette interaction (elles doivent être orientées face à face, dans deux sens opposés). Par ailleurs, les relations d'ordre temporelles sont provisoirement envisagées sous le seul aspect de la succession. La structure praxéologique du procès, notamment quant au statut particulier de la relation d'articulation temporelle, n'en demeure pas moins inchangée.

\section{Agencements rituels}

Jusqu'ici ont été examinées des relations praxéologiques s'inscrivant dans le cadre de procès à finalité essentiellement matérielle, dont les contraintes étaient de caractère physique, fonctionnel: contraintes du corps, du milieu, de l'objet matériel. Si l'on considère à présent les agencements d'actions et d'agents de l'action au sein de procès à dominante rituelle, essentiellement gouvernés par des règles, des normes, des systèmes de valeurs, on constate également l'apparition de chaînes et de suites, spatiales et temporelles, relevant de la composition, de l'ordre et de l'articulation (Tabl. 2). Je ne m'attarderai pas sur leur analyse et me permettrai d'insister sur les chaînes.

Les relations de «proxémie » étudiées par les anthropologues américains à la suite de E.T. Hall (1971) apparaissent, dans cette perspective, comme un cas particulier de contraintes rituelles d'articulation dans l'espace: des chaînes de contact entre les corps, ou des chaînes invisibles de distances à respecter dans diverses circonstances de la vie sociale, variables d'une culture à l'autre.

De leur côté, les distinctions proposées par Mauss (1969) entre rite d'entrée et rites de sortie, par Van Gennep (1969) entre séquences préliminaires, liminaires et postliminaires des rites de passage, constituent des tentatives pour mettre l'accent sur ce qui, dans les actions rituelles, relève d'obligations, non seulement dans la composition temporelle, mais également dans l'ordre temporel; autrement dit, des chaînes rituelles d'ordonnancement temporel.

Pour l'observateur cinéaste, est essentielle la similitude des apparences entre les relations praxéologiques, d'un côté fonctionnelles, que par commodité j’appellerai physiques, de l'autre rituelles. Bien que ces dernières se mettent en scène pour des raisons autres que d'origine physique, elles offrent à qui les observe et les décrit, le spectacle d'agencements de composition, d'ordre et d'articulation qui donnent l'illusion d'être soumis à des contraintes matérielles. De ce point de vue, la séquence d'initiation aux danses de possession du film de Jean Rouch Horendi (1971) - Niger, Songhay-Zerma-, où l'on voit l'initiatrice tenir constamment l'initiée par la ceinture de sa robe tout en dansant, ne se distingue pas fondamentalement du banal pliage de draps de Laveuses.

La rupture essentielle se situe moins entre des procès dont les uns sont mis en forme par des contraintes physiques, les autres par des obligations rituelles, qu'entre des procès quelconques, dont les uns sont soumis à un programme de relations de composition, d'ordre et d'articulation qui offrent une grande rigidité dans l'espace et le temps (chaînes propres aux messes, aux défilés militaires, au travail industriel); les autres manifestent une relative liberté d'agencement (suites propres aux rituels mondains ou ludiques, aux 
tâches domestiques). C'est pourquoi l'observateur cinéaste sera par exemple sensible aux similitudes qu'offrent, dans leur auto-mise en scène, le déroulement d'une messe traditionnelle et celui d'un numéro d'illusionniste, avec son dispositif, sa partenaire et son public. Ces deux sortes de manifestations imposent des contraintes analogues à la mise en scène descriptive du cinéaste.

Peut-être pourrait-on, pour nuancer cette affirmation, hasarder l'hypothèse selon laquelle l'un des traits qui distinguent les procès à dominante rituelle (techniques rituelles) de ceux à dominante physique d'action sur la matière (techniques matérielles), est que les premiers font souvent appel aux écarts obligés entre êtres et choses dans l'espace (chaînes d'articulation invisibles), les seconds aux contacts nécessaires (chaînes) au cours de l'activité productive. Les rites prendraient, en ce sens, le contre-pied des techniques matérielles. Mais ce n'est là qu'une suggestion.

À l'image des relations praxéologiques soumises aux impératifs d'ordre physique, les relations dominées par le rite laissent entrevoir l'existence d'une relative indépendance entre les contraintes de composition, d'ordre et d'articulation. Tantôt tout converge vers la soudure parfaite: le procès, sous ses multiples aspects, est une immense chaîne dans l'espace comme dans le temps (défilé militaire). Tantôt on observe des divergences: la chaîne, localisée dans le temps ou dans l'espace, se limite à l'un des modes de relation (rituels ludiques).

Le parallélisme remarqué entre agencements praxéologiques, les uns physiques, les autres rituels, conduit à examiner de plus près les formes d'intrication entre ces deux niveaux de manifestation d'un procès technique. Ainsi peut être tentée une microanalyse du quotidien qui permette de déceler les gestes rituels, entendus comme des pointes de ritualité dominante émergeant de la ritualité diffuse, et qui prennent place, ici ou là dans le déroulement d'une activité essentiellement soumise à des contraintes de caractère physique. Apparaissent-ils de préférence là où existe une option matérielle, ou plus précisément, une suite matérielle? En d'autres termes, s'insèrent-ils là où cède l'un des maillons de la chaîne fonctionnelle, par exemple, en début ou en fin de procès, lors d'une pause contingente, ou encore, lorsque l'activité matérielle accuse une forme purement répétitive, apparemment gratuite? c'est ce que tendait à laisser penser la méticuleuse finition du pliage des draps par l'une des protagonistes de Laveuses. Telles sont certaines des questions auxquelles on peut espérer à l'avenir pouvoir répondre avec plus d'aisance.

\section{Le jeu des contraintes et des options}

Une première conclusion s'impose: sont rares, non seulement les véritables chaînes, mais plus encore, les procès formés d'un ensemble compact de chaînes, spatiales et temporelles, de composition, d'ordre et d'articulation. Sans doute les rencontre-t-on de préférence dans les manifestations rituelles au programme rigide telles que les cérémonies officielles rigoureusement mises en scène par notre propre société, ou encore dans les activités matérielles aux fortes contraintes de nature biochimique, qui commandent des actes précis, rapides, coordonnés, en raison de la possible dégradation du résultat de l'action (technique de pêche, cuisine, etc.). La crainte de la dégradation du produit de 
l'activité matérielle rejoint alors curieusement la crainte du scandale ou de la sanction qui accompagne la transgression des règles rituelles. Pour l'observateur cinéaste, il s'agit de situations analogues sur le plan scénographique.

Il semble également que parmi les formes de chaînes possibles, celles de composition spatiale soient les plus fréquemment rencontrées. Cela se comprend aisément: la plupart des activités humaines s'accomplissent grâce à la présence complémentaire d'éléments humains et matériels (le « quoi » de la composition) dont la fonction est indispensable, la forme - ou la nature-, irremplaçables pour diverses raisons. Toutefois, ces fréquentes contraintes de composition sont compensées par une relative liberté dans les manières d'agencer les actions et les agents humains ou matériels de l'action (le « comment » de l'ordre et de l'articulation), dans l'espace et dans le temps. Sans cette soupape de sûreté qu'est une relative souplesse dans le comment de l'action, les programmes humains se distingueraient peu des programmes instinctifs propres aux animaux.

Dans l'expérience quotidienne, telle ou telle manifestation de l'activité nous semble totalement obéir à la contrainte ou, au contraire, être librement conduite. Cela tient, par exemple, à ce que dans un cas, elle obéit à une chaîne temporelle, dans l'autre, elle est truffée de pauses. Or, bien souvent, ce n'est là qu'une illusion: une option est masquée par le continuum technico-rituel, une chaîne se glisse la ou on ne l'attendait pas. Aussi n'est-il pas inutile de pouvoir repérer avec une relative précision, au sein d'un procès dans lequel, au premier abord, tout semble strictement programmé par la contrainte, le moment souvent fugace, imperceptible, où surgit une option, marque subtile de la liberté; inversement, de pouvoir discerner ici ou là, dans un procès où tout semble librement agencé à première vue, les marques ponctuelles de la contrainte: par exemple, les quelques brèves chaînes d'articulation temporelle qui s'insèrent dans la longue suite articulatoire des gestes de fabrication du vannier de La Charpaigne.

Une analyse fine des contraintes et des options, tenant compte simultanément des divers modes d'agencement, devrait permettre d'éclairer davantage les activités les plus banales de la vie quotidienne - notamment domestiques, trop souvent considérées comme des chaînes strictement programmées. Elle ferait apparaître la part importante qu'y jouent les options, voire les inventions, aux différents niveaux de relations praxéologiques, de même que la maîtrise, par les agents, des intrications entre les tâches.

Une nouvelle étape de la recherche est engagée, au cours de laquelle seront examinés de plus près les principes de coordination entre les modes d'agencement praxéologiques. Car en dépit des possibilités d'indépendance entre les modes, entre les registres spatial et temporel, que l'on découvre en se plaçant dans la perspective d'une praxéologie générale, existent, au sein de la plupart des procès, des relations de forte implication simple ou réciproque, entre la composition, l'ordre et l'articulation. Contraintes, options, s'emboîtent alors les unes dans les autres, se commandent les unes les autres, à divers niveaux de l'action. Existeraient ainsi des contraintes ou des options dominantes, en ce sens qu'elles commanderaient en partie ou en totalité le développement d'un procès. La question posée serait alors: lequel de l'ordre, de la composition ou de l'articulation commande les deux autres?

Le cas du banc à planer du vannier de La Charpaigne est un exemple de contrainte dominante. La présence d'un élément de composition spatiale obligé, le vannier en posture debout, commande celle d'un élément complémentaire, le banc à planer, support matériel extérieur au corps de l'agent. Tous deux forment ensemble une chaîne spatiale de composition qui commande à son tour une chaîne d'ordre également spatiale. En effet, 
le vannier doit être constamment orienté dans le prolongement de son banc à planer au cours des différentes étapes de la fabrication. Pour un observateur cinéaste, la connaissance d'une telle dominance est décisive.

\section{Cinéma et arthrologie}

Par bien des côtés, le système d'analyse praxéologique dont viennent d'être esquissées les grandes lignes peut paraître aller de soi. C'est que chacun des modes de relations, ou d'agencements, pris séparément, semble extrêmement familier à qui s'est penché un jour ou l'autre, en technologue ou en ritologue, sur le problème de la description des activités humaines. On ne saurait cependant en dire autant de ces mêmes modes pris ensemble et considérés sous l'angle de leur coordination, car la dissociation des diverses formes de relations praxéologiques, au sein d'un même procès, exige l'abandon d'habitudes de pensée très anciennes. Au cours de l'analyse, le chercheur est bien souvent tenté, à son insu même, soit d'amalgamer ordre et composition, ou bien ordre et articulation, soit de négliger l'articulation au profit de l'ordre et de la composition, etc.

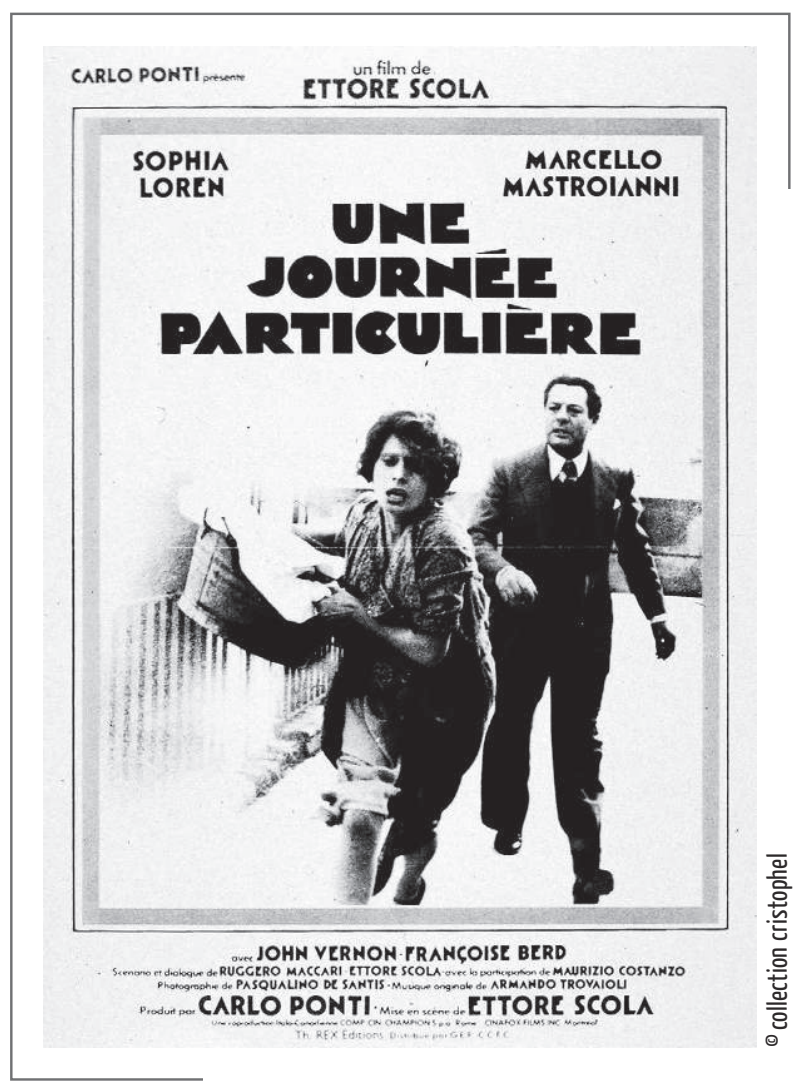

Les catégories de la logique héritée des anciens Grecs, celles de la grammaire également, nous ont familiarisés avec la composition et l'ordre; ces deux formes de relation sont à la base de la plupart des classifications ${ }^{10}$. La prédominance du langage oral, puis de l'écriture comme techniques d'expression, en est en grande partie responsable. Et lorsque l'homme dispose, pour explorer les formes de l'action, de l'observation directe, les articulations entre les actions, ou entre les agents de l'action, souvent fugaces ou estompées, passent inaperçues. Elles exigeraient, pour être recueillies, de voir leurs manifestations indéfiniment répétées, ce que, précisément, ne permet pas l'observation directe. Aussi n'est-ce pas l'effet du hasard si cette forme de relations praxéologiques, qui relève d'une arthrologie des êtres en mouvement et en transformation, n'est devenue saisissable qu'avec l'introduction de la cinématographie dans l'appareil de recherche de l'anthropologue. On ne s'étonnera guère, en ce cas, de ce que le créateur de la notion de « chaîne opératoire ", André Leroi-Gourhan (1948), sensible à la fluidité du continuum technico-rituel, a été par ailleurs l'un des instigateurs de l'utilisation du cinéma en ethnologie.

Mettre en lumière les relations d'articulation à partir de l'usage de la cinématographie n'est pas sans incidences sur la manière de concevoir les relations de composition. Ainsi, les traits fonctionnels de composition spatiale (agents humains, instruments, objets) constituant 
à l'origine les termes de toute relation d'articulation, par jonction ou séparation, on est en droit de supposer qu'ils forment des unités discrètes de composition, physiquement séparables. Or, la cinématographie nous enseigne l'impossibilité de séparer parfois, au plan des faits sensibles, ce que l'écriture nous a appris à dissocier mentalement: par exemple, l'agent et l'instrument corporel réunis en un même corps, tels l'artisan vannier et sa main qui travaille le bois. Aussi les points de rupture ne se situent-ils pas nécessairement entre des éléments ponctuels, mais entre des regroupements compacts d'éléments de composition, formant un continuum spatial d'agents humains et matériels.

Dans le temps également, l'accent mis sur les relations d'articulation introduit une forme de découpage du procès qui peut ne pas coïncider avec le découpage des actions envisagées du strict point de vue de la composition. C'est ainsi qu'une chaîne d'articulation temporelle recouvrira parfois une longue succession d'opérations.

Il existe donc une relative concurrence entre les manières de décrire un procès, selon qu'elles prennent appui sur les relations de composition ou d'articulation. Une telle concurrence semble renvoyer à deux modes de pensées dont l'emploi de la cinématographie révèle l'incompatibilité partielle. 


\section{NOTES DE L'ARTICLE}

1. La Charpaigne traite de la fabrication d'une vannerie en bois de noisetier - ou coudrier - utilisé pour le transport vers l'étable des aliments destinés au bétail. Le film a été tourné dans le village de Lignerolles, situé en région forestière au nord-est de la Bourgogne, dans le Châtillonnais.

2. Laveuses est consacré à la description des différentes phases de l'entretien du linge par les femmes du même village de Lignerolles qui continuent à utiliser le lavoir communal même en hiver.

3. La distinction d'ordre praxéologique entre « activité » et «procès » peut être rapprochée de celle proposée par Marx, dans Le Capital entre « procès de travail » et " procès de production », ce dernier ne nécessitant pas la participation directe de l'homme pour s'accomplir.

4. Techniques de musculation fut tourné dans un centre sportif de la région parisienne, pendant une année entière, à raison d'une séance de tournage par semaine, auprès de jeunes gymnastes amateurs s'adonnant à la musculation libre.

5. Le Coiffeur itinérant a pour sujet la séance de coupe de cheveux par un coiffeur, lors de son passage mensuel dans le village de Lignerolles. Elle a lieu dans le café-épicerie du village, dont la patronne est l'une des clientes du coiffeur. Cette technique du corps est l'occasion de rencontres régulières entre les habitants qui trinquent, échangent des nouvelles, plaisantent; en un mot elle sert de support à un rite profane.

6. La question des éléments du milieu marginal, par rapport à ceux du milieu efficient, fait l'objet d'un traitement particulier dans Cinéma et anthropologie (1982).

7. Il est plusieurs manières de considérer cette suite formelle, selon la perspective théorique et méthodologique dans laquelle on se place. On peut y voir, par exemple, l'expression d'un niveau du « fait » par opposition à la « tendance » fonctionnelle (perspective évolutionniste développée par A. Leroi-Gourhan); celles d'une «variante » dans la combinaison des traits possibles (perspective structuraliste); enfin celle d'une «transformation », relevant de la performance individuelle plus que de la compétence (perspective générativiste), cette dernière interprétation combinant certains aspects propres aux deux autres.

8. Des transgressions de cette sorte, comme celles qui affectent la composition et l'articulation, sont bien souvent à la base des gags les plus efficaces du cinéma de fiction. Conduisant les protagonistes, soit à l'échec, soit à une réussite inattendue, elles sont dans les deux cas source de rire.

9. Dans cette séquence, le caractère très apparent de la chaîne spatiale (le contact obligé des mains avec le drap), allié au fait que les deux plieuses ne marquent effectivement pas de pause, créent ensemble l'illusion d'une chaîne temporelle d'articulation.

10. Tout peut même être traduit en termes de composition. Rien n'empêche par exemple, de réduire la tripartition proposée dans cet exposé (composition, ordre, articulation) à la seule composition, dans le souci d'une formalisation plus poussée. Les intervalles et les pauses s'exprimeraient alors en termes d'absence d'éléments et d'actions. Cependant cette économie logique présenterait un faible intérêt sur le plan de l'observation cinématographique. De plus, on ne ferait que déplacer au niveau des attributs de la composition, la complexité des catégories d'articulation et d'ordre. 


\section{RÉFÉRENCES}

France, Cl. de 1982 Cinéma et anthropologie. Paris: Éditions de la Maison des sciences de l'homme.

Hall, E. T. 1971 La Dimension cachée. Paris: Seuil.

Leroi-Gourhan, A. 1948 Cinéma et sciences humaines. Le film ethnologique existe-t-il? La revue de géographie humaine et d'ethnologie, $3: 42-51$.

- 1964 Le Geste et la parole. 1, Techniques et langage. Paris: Albin Michel.

- 1965 Le Geste et la parole. 2, La mémoire et les rythmes. Paris: Albin Michel.

Mauss, M. 1969 Fuvres, 2. Paris: Minuit.

Van Gennep, A. 1969 Les Rites de passage. Paris/La Haye: Mouton et Éditions de la Maison des sciences de

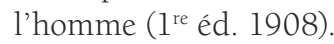

- Filmographie

France, Cl. de 1969 La Charpaigne. 16 mm, noir et blanc, 30 min. Dépôt Paris: Comité du film ethnographique.

— 1970 Laveuses. 16 mm, couleur, 30 min. Dépôt Paris: Comité du film ethnographique.

— 1972 Le Coiffeur itinérant. 16 mm, couleur, 25 min. Dépôt Paris: Comité du film ethnographique.

— 1973 Techniques de musculation. 16 mm, couleur, 120 min. Dépôt Paris: Comité du film ethnographique.

-1980-82 Bazar. Vidéo 3/4 de pouce, noir et blanc, épreuves 200 min. Dépôt Université de Paris X Nanterre: Formation de recherche cinématographique.

Rouch, J. 1971 Horendi. 16 mm, couleur, 90 min. Dépôt Paris: Comité du film ethnographique.

Scola, E. 1977 Une journée particulière. 35 mm, couleur, 101 min.

\section{NOTES ET RÉFÉRENCES DE L'INTRODUCTION}

Toutes les illustrations sont la propriété de l'auteur de l'article à l'exception de l'image d'ouverture, scène extraite d'Une journée particulière d'Ettore Scola et de l'affiche du film, toutes deux reproduites avec l'aimable autorisation de Serge Darmon, collection christophel, www.collectionchristophel.fr.

Poe, E. A. La Lettre volée (The Purloined Letter). The Gift : A Christmas and New Year's Present, automne 1844. 